\title{
Factors associated with elevated intraocular pressure in eyes with iris melanoma
}

Carol L Shields, Miguel A Materin, Jerry A Shields, Eric Gershenbaum, Arun D Singh, Andrew Smith

\begin{abstract}
Aim-To identify clinical factors associated with secondary elevated intraocular pressure (IOP) in eyes with iris malignant melanoma
\end{abstract}

Methods-A retrospective case series of 169 consecutive patients with microscopically confirmed iris malignant melanoma. The main outcome measure was the presence of tumour induced secondary elevated IOP. Cox proportional regression models were used to calculate the relation of clinical features to elevated IOP.

Results-Of 169 patients with microscopically proved iris melanoma, $50(30 \%)$ presented with tumour induced secondary elevated IOP. The mean pressure in those eyes with elevated IOP at diagnosis was 33 mm $\mathrm{Hg}$ (median $31 \mathrm{~mm} \mathrm{Hg}$, range 23-65 mm Hg). The tumour configuration was nodular in 23 (46\%) and diffuse in 27 (54\%) with a mean base dimension of $7.4 \mathrm{~mm}$ and thickness of $2.0 \mathrm{~mm}$. Invasion of the angle structures by melanoma seeds was visible for a mean of 7 clock hours (median 7, range 0-12 clock hours). The mechanism of elevated IOP was judged to be outflow obstruction from tumour invasion into the trabecular meshwork. There were no cases of neovascular glaucoma. The tumour was ultimately managed with enucleation in 30 patients $(60 \%)$, local resection (iridectomy, iridocyclectomy, or iridocyclogoniectomy) in $11(22 \%)$, and plaque radiotherapy in five $(\mathbf{1 0 \%})$. In four cases $(8 \%)$, observation of cytologically low grade tumour was the patient's preference. Using multivariate analysis, the clinical factors at initial evaluation associated with tumour induced secondary elevated IOP from iris melanoma included increasing extent of tumour seeding in the anterior chamber angle $(p=0.01)$ and poor visual acuity at presentation $(\mathbf{p}=0.02)$.

Conclusions-Microscopically confirmed iris melanoma demonstrates tumour related elevated IOP in $30 \%$ cases at the time of presentation, usually secondary to tumour involvement of the trabecular meshwork obstructing aqueous outflow. Enucleation is necessary in the majority of these patients $(60 \%)$ as opposed to those cases without elevated intraocular pressure $(18 \%)$.

(Br f Ophthalmol 2001;85:666-669)
Iris melanomas represent only $2 \%$ to $3 \%$ of all uveal melanomas. ${ }^{1-4}$ The clinical features suggestive of iris melanoma are variable and include a documented enlarging melanocytic iris mass of nodular or flat configuration with or without seeding of the tumour onto the adjacent iris and into the anterior chamber angle..$^{5-7}$ Most iris melanomas are discovered at a relatively small size and are of low grade cell type; hence, a favourable prognosis is usually anticipated with only $5 \%$ metastasis at 10 years. ${ }^{1-489}$ Recently published data have revealed important risk factors for metastatic disease from iris melanoma including older age at diagnosis, tumour involvement in the anterior chamber angle, elevated intraocular pressure (IOP), and extraocular extension of tumour. ${ }^{4}$

The association of elevated IOP with intraocular tumours is well recognised. In 1987, Shields and associates reported that $5 \%$ of 2704 eyes with intraocular tumours demonstrated elevated IOP. ${ }^{10}$ Secondary IOP elevation was found in $7 \%$ of eyes with clinically diagnosed iris melanoma, $17 \%$ of eyes with ciliary body melanoma, and $2 \%$ of eyes with choroidal melanoma. Other tumours that induce secondary elevated IOP include uveal metastasis, retinoblastoma, and medulloepithelioma. With regard to retinoblastoma, elevated IOP has been found to promote risk for optic nerve invasion of tumour and subsequent tumour metastasis. ${ }^{11}$ Similarly, elevated IOP has been found to be a risk for iris melanoma metastasis. ${ }^{4}$ In this report, we more closely investigate clinical factors related to elevated IOP in eyes with iris melanoma. The mechanisms of elevated IOP in these eyes are discussed.

\section{Patients and methods}

The clinical records of all patients with the diagnosis of iris malignant melanoma managed on the Ocular Oncology Service at Wills Eye Hospital, Philadelphia, between June 1974 and June 1999 were reviewed. Only those patients with microscopically confirmed (histopathologically and/or cytopathologically) melanoma that arose in the iris were included in this study. Tumours that originated in the ciliary body with secondary iris extension were excluded. Clinical data were gathered regarding features of the patient, the eye, the tumour, the mechanisms of elevated IOP, and clinical management and follow up. A separate analysis of this database regarding melanoma related metastasis will be published elsewhere. ${ }^{4}$ The clinical data in this analysis were analysed with
Accepted for publication 12 January 2001 
regard to the finding of ipsilateral elevated IOP greater than $22 \mathrm{~mm} \mathrm{Hg}$ and at least $5 \mathrm{~mm}$ greater than the contralateral eye.

The data included patient features at initial examination such as age, race, and sex. The ocular data included affected eye, best corrected Snellen visual acuity, IOP $(\mathrm{mm} \mathrm{Hg}$ ), and iris colour. The tumour data included predominant quadrant location, clock hour meridian, number of clock hours affected by tumour (1-12 hours), anteroposterior epicentre and margins (pupil, midzone, root, angle, diffuse), configuration (nodular, flat), basal dimension (millimetres), thickness (millimetres), tumour colour (brown, yellow), and surface features (irregular, smooth). Other clinical features included clock hour extent of adjacent iris seeds (0-12 hours), tumour satellites, ectropion iridis, corectopia, episcleral sentinel vessel, feeder iris vessel, intrinsic tumour vessels, clock hour extent of anterior chamber angle seeds, anterior chamber features, secondary cataract, and extraocular extension. The mechanisms of elevated IOP were determined. The tumour management after referral to us (observation, iridectomy, iridocyclectomy, iridogoniocyclectomy, plaque radiotherapy, enucleation) was assessed.

Table 1 A comparison of the clinical features of 169 consecutive eyes with iris melanoma with and without secondary elevated intraocular pressure (IOP)

\begin{tabular}{|c|c|c|}
\hline & $\begin{array}{l}\text { Eyes with elevated IOP } \\
(n=50)\end{array}$ & $\begin{array}{l}\text { Eyes without elevated IOP } \\
(n=169)\end{array}$ \\
\hline \multicolumn{3}{|l|}{ Patient age (years) } \\
\hline \multicolumn{3}{|l|}{ Visual acuity } \\
\hline $20 / 20-20 / 40$ & $32(64 \%)$ & $112(94 \%)$ \\
\hline $20 / 50-20 / 100$ & $10(20 \%)$ & $6(5 \%)$ \\
\hline $20 / 200$ or worse & $6(12 \%)$ & $1(<1 \%)$ \\
\hline unavailable & $2(4 \%)$ & 0 \\
\hline \multicolumn{3}{|l|}{ Iris colour } \\
\hline blue & $43(86 \%)$ & $100(84 \%)$ \\
\hline green & $7(14 \%)$ & $10(8 \%)$ \\
\hline brown & $0(0 \%)$ & $9(8 \%)$ \\
\hline \multicolumn{3}{|l|}{ Intraocular pressure $(\mathrm{mm} \mathrm{Hg})$} \\
\hline \multicolumn{3}{|l|}{ Tumour size (No of clock hours) } \\
\hline mean (median, range) & $5(4,1-12)$ & $3(2,1-12)$ \\
\hline \multicolumn{3}{|l|}{ Tumour base (mm) } \\
\hline mean (median, range) & $7(6,1-13)$ & $6(5,1-12)$ \\
\hline \multicolumn{3}{|l|}{ Tumour thickness (mm) } \\
\hline mean (median, range) & $2(2,1-4)$ & $2(2,1-4)$ \\
\hline \multicolumn{3}{|l|}{ Tumour configuration } \\
\hline nodule & $23(46 \%)$ & $98(82 \%)$ \\
\hline flat & $27(54 \%)$ & $21(18 \%)$ \\
\hline \multicolumn{3}{|l|}{ Tumour colour } \\
\hline brown & $39(78 \%)$ & $85(71 \%)$ \\
\hline yellow & $11(22 \%)$ & $34(28 \%)$ \\
\hline \multicolumn{3}{|l|}{ Tumour seeding iris (No of clock hours) } \\
\hline \multicolumn{3}{|c|}{ Tumour seeding angle (No of clock hours) } \\
\hline mean (median, range) & $7(7,0-12)$ & $3(1,0-12)$ \\
\hline \multicolumn{3}{|l|}{ Associated findings } \\
\hline ectropion iridis & $23(46 \%)$ & $52(44 \%)$ \\
\hline corectopia & $29(58 \%)$ & $75(63 \%)$ \\
\hline prominent episcleral vessel & $17(34 \%)$ & $26(22 \%)$ \\
\hline feeding tumour vessel & $13(26 \%)$ & $29(24 \%)$ \\
\hline intrinsic tumour vessel & $17(34 \%)$ & $55(46 \%)$ \\
\hline hyphaema & $4(8 \%)$ & $12(10 \%)$ \\
\hline cell and flare & $3(6 \%)$ & $4(3 \%)$ \\
\hline secondary cataract & $5(10 \%)$ & $18(15 \%)$ \\
\hline \multicolumn{3}{|l|}{ Tumour management } \\
\hline enucleation & $30(60 \%)$ & $21(18 \%)$ \\
\hline local resection $\star$ & $11(22 \%)$ & $91(76 \%)$ \\
\hline plaque radiotherapy & $5(10 \%)$ & $4(3 \%)$ \\
\hline observationt & $4(8 \%)$ & $3(2 \%)$ \\
\hline
\end{tabular}

^Local resection $=$ iridectomy, iridocyclectomy, or iridocyclogoniectomy.

†Observation $=$ in all cases the tumour was cytologically low grade and observation was patient's preference.
STATISTICAL ANALYSIS

The relation of each individual clinical variable to the presence of tumour induced elevated IOP was analysed by a series of univariate Cox proportional hazards regressions. The correlation among the variables was determined by using Pearson correlations. All variables were analysed as discrete variables except for patient age, IOP, tumour base, tumour thickness, and number of clock hours affected by tumour or seeds, which were analysed as continuous variables. The variables that were significant at a univariate level $(p<0.05)$ were entered into a stepwise regression analysis. For variables that showed a high degree of correlation, only one variable from the set of associated variables was entered at a time in subsequent multivariate models. A final multivariate model fitted variables that were identified as significant predictors $(p<0.05)$ in the stepwise model as well as variables deemed clinically important for the outcome of elevated IOP.

\section{Results}

Of 169 patients with microscopically confirmed iris melanoma, $50(30 \%)$ had evidence of elevated IOP at the time of presentation to our service. A summary of the clinical features of the group of eyes with elevated IOP versus the group of eyes without elevated IOP is provided in Table 1 (Fig 1) In the group with elevated IOP, 29 of the 50 patients $(58 \%)$ had pressure $30 \mathrm{~mm} \mathrm{Hg}$ or greater.

By univariate analysis, the most important clinical features associated with elevated IOP were increasing size of tumour base $(\mathrm{p}<0.0001)$, tumour epicentre at iris root $(p<0.0001)$, flat tumour configuration $(\mathrm{p}<0.0001)$, increasing angle seeding by tumour $(\mathrm{p}<0.0001)$, increasing
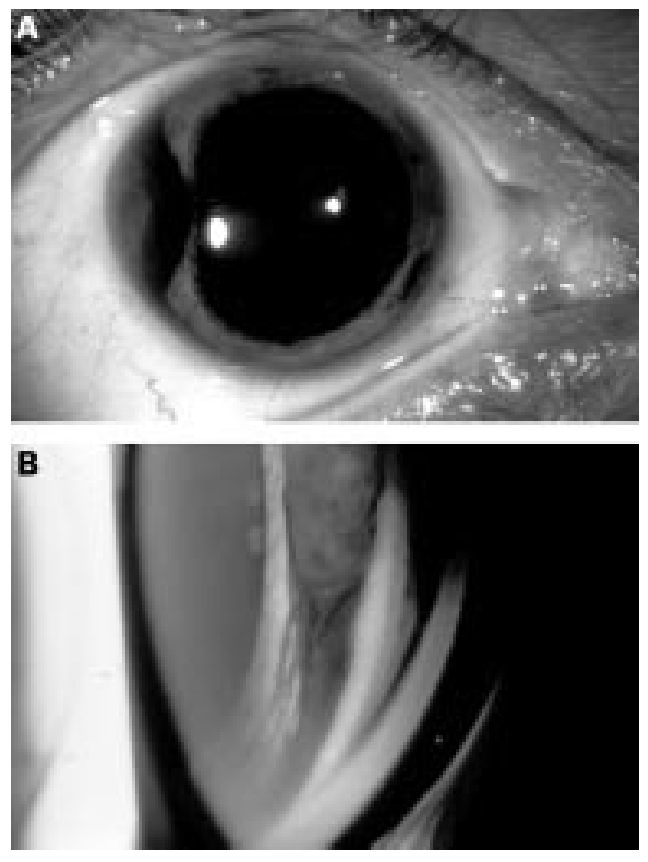

Figure 1 Iris melanoma with advanced tumour seeding in the angle and intraocular pressure of $36 \mathrm{~mm} \mathrm{Hg}$. (A) Slit lamp appearance of the fusiform pigmented mass involving the temporal portion of the iris. (B) Gonioscopic appearance of the mass with a tail of seeding into the angle for 12 clock hours, completely infiltrating the trabecular meshwork. 
Table 2 Univariate Cox proportional hazards analyses of clinical factors predictive of elevated intraocular pressure (>22 $\mathrm{mm} \mathrm{Hg}$ ) in 169 eyes with microscopically proved iris melanoma

\begin{tabular}{|c|c|c|c|c|}
\hline Clinical feature & $p$ Value & Relative risk & $95 \%$ CIt & {$[c, d]$} \\
\hline $\begin{array}{l}\text { Tumour base (No clock hours) } \\
\text { Increasing }\end{array}$ & $<0.0001$ & 1.17 & $1.08,1.25$ & c \\
\hline $\begin{array}{l}\text { Tumour epicentre } \\
\text { Iris root } v \text { pupillary margin }\end{array}$ & $<0.0001$ & 1.67 & $1.34,2.08$ & $\mathrm{~d}$ \\
\hline $\begin{array}{l}\text { Tumour shape } \\
\text { Flat } v \text { nodule }\end{array}$ & $<0.0001$ & 4.63 & $2.55,8.40$ & $\mathrm{~d}$ \\
\hline $\begin{array}{l}\text { Angle seeds (No of clocks hours) } \\
\text { Increasing hours }\end{array}$ & $<0.0001$ & 1.16 & $1.09,1.24$ & $\mathrm{c}$ \\
\hline $\begin{array}{l}\text { Iris seeds (No of clock hours) } \\
\text { Increasing }\end{array}$ & 0.0001 & 1.11 & $1.05,1.17$ & $\mathrm{c}$ \\
\hline $\begin{array}{l}\text { Visual acuity at diagnosis } \\
<20 / 20 v 20 / 20^{\star}\end{array}$ & 0.0003 & 1.13 & $1.06,1.21$ & $\mathrm{~d}$ \\
\hline $\begin{array}{l}\text { Tumour base }(\mathrm{mm}) \\
\text { Increasing }\end{array}$ & 0.0005 & 1.15 & $1.06,1.24$ & c \\
\hline $\begin{array}{l}\text { Posterior tumour margin } \\
\text { Iris root/angle } v \text { midzone }{ }^{\star}\end{array}$ & 0.002 & 1.80 & $1.24,2.60$ & $\mathrm{~d}$ \\
\hline $\begin{array}{l}\text { Angle seeds } \\
\text { Present } v \text { absent }{ }^{\star}\end{array}$ & 0.009 & 1.84 & $1.17,2.90$ & $\mathrm{~d}$ \\
\hline $\begin{array}{l}\text { Episcleral vessel } \\
\text { Present } v \text { absent }{ }^{\star}\end{array}$ & 0.03 & 1.92 & $1.07,3.47$ & $\mathrm{~d}$ \\
\hline
\end{tabular}

†CI $=$ confidence interval.

$\mathrm{c}=$ continuous variable, $\mathrm{d}=$ discrete variable.

${ }^{\star}$ Reference variable.

Table 3 Multivariate Cox proportional hazards analyses of clinical factors predictive of elevated intraocular pressure (>22 $\mathrm{mm} \mathrm{Hg}$ ) in 169 eyes with microscopically proved iris melanoma

\begin{tabular}{lllll}
\hline Clinical feature & $p$ Value & Relative risk & $95 \%$ CI† & {$[c, d]$} \\
\hline $\begin{array}{l}\text { Angle seeds (No of clock hours) } \\
\text { Increasing hours }\end{array}$ & 0.01 & 1.4 & $1.06,1.74$ & $\mathrm{c}$ \\
$\begin{array}{l}\text { Visual acuity at diagnosis } \\
<20 / 20 v 20 / 20^{\star}\end{array}$ & 0.02 & 1.4 & $1.05,1.81$ & $\mathrm{~d}$ \\
\hline$+\mathrm{CI}=$ confidence interval. & & & \\
$\mathrm{c}=$ continuous variable, $\mathrm{d}=$ discrete variable. \\
$\star$
\end{tabular}

iris seeding by tumour $(\mathrm{p}=0.0001)$, and visual acuity less than 20/20 ( $\mathrm{p}=0.0003$ ) (Table 2). By multivariate analysis, the most important group of clinical features predictive of elevated IOP included increasing involvement of the angle with tumour seeds $(\mathrm{p}=0.01)$ and visual acuity less than 20/20 $(\mathrm{p}=0.02)$ (Table 3$)$.

\section{Discussion}

The clinical features of iris melanoma can vary from case to case. Most often this tumour presents in individuals with blue irides as a pigmented mass at or below the horizontal meridian of the iris. ${ }^{4-7}$ At the time of clinical

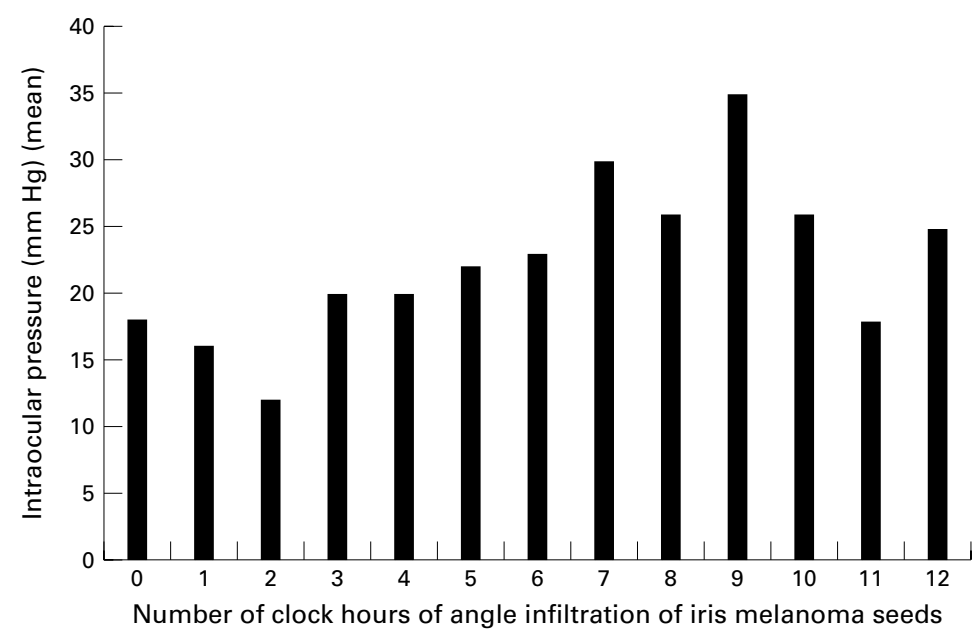

Figure 2 Relation of the clock hour extent of iris melanoma seeding in the anterior chamber angle with intraocular pressure in 169 consecutive cases. diagnosis, the average tumour size has been reported at $6 \mathrm{~mm}$ in base and $2 \mathrm{~mm}$ in thickness. ${ }^{4}$ Associated features include ectropion iridis $(44 \%)$, corectopia $(62 \%)$, hyphaema (9\%), enlarged vessels feeding the tumour $(25 \%)$, intrinsic tumour vessels (43\%), tumour induced cataract $(14 \%)$, and elevated IOP $(30 \%) .^{4}$ In one series of clinical diagnosed iris melanoma, associated elevated IOP was found in $7 \%$ of patients ${ }^{10}$ whereas in those cases that come to histopathology, 30\% had elevated IOP. ${ }^{4}$ Many of these associated features can also be found with the more common benign iris naevus and differentiation can be challenging and depends on many factors, most importantly the documentation of tumour growth..$^{5-7} 12$

Previous reports on iris melanoma have focused mostly on the histopathological features of this tumour. ${ }^{8913}{ }^{14}$ Few clinicians have had experience with a large clinical series of patients with iris melanoma. ${ }^{134}$ We recently published our experience with 169 patients with microscopically proved iris melanoma and identified clinical factors predictive of metastases ${ }^{4}$ one of which was elevated IOP. In this report we further investigate the group of patients with elevated IOP. The median age at presentation was 53 years for those with elevated IOP versus 41 years for those without elevated IOP (Table 1). The median number of clock hours affected by tumour was 6 in those with elevated IOP versus 2 in those without elevated IOP. The median number of clock hours of angle seeding was 7 in those with elevated IOP versus 1 in those without elevated IOP. Additionally, visual acuity at presentation was $20 / 200$ or poorer in $12 \%$ of those with elevated IOP versus less than $1 \%$ of those without elevated IOP. It is thus apparent that most patients who develop secondary elevated IOP have larger tumours with more advanced features as indicated in the univariate analysis (Table 2). In the multivariate analysis, increased seeding of melanoma into the anterior chamber angle and poor visual acuity were the two most important factors associated with secondary elevated IOP (Table 3). Patients with less than $20 / 20$ visual acuity were more likely to have associated elevated intraocular pressure than those with $20 / 20$ visual acuity. It is difficult to explain this finding, but it could be related to various abnormalities including glaucoma induced scotoma, lens tilt from the iris mass, ocular surface irritation from elevated intraocular pressure, corneal oedema, or others.

Our study indicates that the most common mechanism of elevated IOP is tumour infiltration of the trabecular meshwork with outflow obstruction. None of our patients had neovascular glaucoma. There may be other mechanisms involved in the production of elevated IOP as 15 of 73 patients (20\%) had elevated IOP with no visible angle seeds or neovascularisation of the iris. In general, the greater the clock hour involvement with angle seeds, the greater the IOP. Patients with 6 or more clock hour infiltration of tumour seeds showed a mean IOP of $23 \mathrm{~mm}$ or greater (Fig 2). The 
presence of angle seeds was not completely correlated with elevated IOP as 16 of 35 patients (46\%) with 12 clock hours of tumour seeding into the angle had IOP less than 23 $\mathrm{mm} \mathrm{Hg}$.

The purpose of this report was to identify clinical features associated with elevated IOP in eyes with iris melanoma. We did not assess the treatment of elevated IOP, in particular, as our primary goal was treatment of the malignancy. However, we can generally state our philosophy regarding management of melanocytic iris tumours with elevated IOP. In most instances, the differentiation of iris melanoma from benign iris naevus must first be made before decisions regarding elevated IOP are addressed. If the tumour is small (1-2 clock hours at the base) and there is no evidence of growth or angle seeding, then observation of the tumour and conservative treatment of the elevated IOP with topical medications is a reasonable first step. Further therapeutic options include oral medications and cautious laser trabeculoplasty at uninvolved trabecular meshwork sites or ciliary body ablation. Trabeculectomy or other open eye procedures should be avoided because of the risk for extraocular extension, should the tumour eventually grow. ${ }^{15}$ In those eyes with large iris melanoma (3 clock hours or greater), tumours with substantial angle seeding, or tumours with documented tumour growth, primary management of the tumour should be performed first and then reassessment of the elevated IOP later. Management options for iris melanoma include complete local resection by iridectomy, iridocyclectomy, or iridogoniocyclectomy techniques, plaque radiotherapy, or enucleation. ${ }^{16}{ }^{17}$ Local resection is planned for smaller tumours with minimal, if any, tumour seeding. ${ }^{16}$ Custom designed plaque radiotherapy is considered for iris tumours too large for local resection, usually in older patients. ${ }^{17}$ It should be realised that the majority of patients $(60 \%)$ with iris melanoma and elevated intraocular pressure eventually require enucleation. If non-enucleation measures are employed, then conservative management of the elevated IOP with topical or oral medications, laser trabeculoplasty, or ciliary body ablation is advised and trabeculectomy and other open eye procedures should be avoided. From our series, enucleation was more commonly employed for iris melanoma with elevated IOP $(60 \%)$ than those without elevated IOP (18\%).

Our results should be interpreted with caution. This series may be biased towards more advanced iris melanoma, reflecting our tertiary referral service. We may be more likely to be referred patients with complications of iris melanoma, such as secondary elevated IOP. Additionally we did not assess treatment of secondary elevated IOP, but only assessed factors associated with elevated IOP.

In summary, secondary elevated IOP is found in $30 \%$ of eyes with microscopically proved iris melanoma. The most common mechanism of elevated IOP is tumour infiltration of the trabecular meshwork and not angle closure or neovascular glaucoma as seen with posterior uveal melanoma. ${ }^{10}$ Factors associated with elevated IOP in eyes with iris melanoma include poor visual acuity at presentation and tumour seeding into the anterior chamber angle. These features should be assessed in all patients with iris melanoma to evaluate for hidden tumour in the angle, most importantly, and also to anticipate elevated IOP.

Support provided by the Paul Kayser International Award of Merit in Retina Research, Houston, TX, USA (J Shields), Macula Foundation, New York, NY, USA (C Shields), and the Eye Tumor Research Foundation, Philadelphia, PA, USA (C Shields).

1 Jensen OA. Malignant melanomas of the human uvea. Recent follow-up of cases in Denmark, 1943-1952. Acta Ophthalmol 1970;48:1113-28.

2 Geisse LJ, Robertson DM. Iris melanomas. Am f Ophthalmol 1985;99:638-48.

3 Batioglu F, Gunalp I. Malignant melanomas of the iris. fap f Ophthalmol 1998;42:281-5.

f Ophthalmol 1998; $42: 281-5$.
4 Shields CL, Shields JA, Materin M, et al. Iris melanoma: risk Shields CL, Shields JA, Materin M, et al. Iris melanoma: risk
factors for metastasis in 169 consecutive patients. Ophthalmology (in press).

5 Shields JA, Shields CL. Melanocytic tumors of the iris stroma. In: Intraocular tumors. A text and atlas. Philadelphia: WB Saunders, 1992:61-84

6 Shields JA, Shields CL. Melanocytic tumors of the iris stroma. In: Atlas of intraocular tumors. Philadelphia: Lippincott, Williams, and Wilkins, 1999:17-42.

7 Shields JA, Sanborn GE, Augsburger JJ. The differential diagnosis of malignant melanoma of the iris. A clinical study of 200 patients. Ophthalmology 1983;90:716-20.

8 Sunba MS, Rahi AHS, Morgan G. Tumors of the anterior uvea. I. Metastasizing malignant melanoma of the iris. Arch Ophthalmol 1980;98:82-5.

9 Rones B, Zimmerman LE. The prognosis of primary tumors of the iris treated by iridectomy. Arch Ophthalmol 1958:60:193-205.

10 Shields CL, Shields JA, Shields MB, et al. Prevalence and mechanisms of secondary intraocular pressure elevation in eyes with intraocular tumors. Ophthalmology 1987;94:83946.

11 Shields CL, Shields JA, Baez K, et al. Optic nerve invasion of retinoblastoma. Metastatic potential and clinical risk factors. Cancer 1994;73:692-8.

12 Territo C, Shields CL, Shields JA, et al. Natural course of melanocytic tumors of the iris. Ophthalomology 1988;95: 1251-5.

13 Arentsen JJ, Green WR. Melanoma of the iris. Ophthalmic Surg 1975;6:23-37.

14 Jakobiec FA, Silbert G. Are most iris "melanomas" really nevi? A clinicopathologic study of 189 lesions. Arch Ophthalmol 1981;99:2117-32.

15 Grossniklaus HE, Brown RH, Stulting RD, et al. Iris melanoma seeding through a trabeculectomy site. Arch Ophthalmol 1990;108:1287-90.

16 Shields JA, Shields CL. Surgical approach to lamellar sclerouvectomy for posterior uveal melanomas. The $1986 \mathrm{Sch}-$ ouvectomy for posterior uveal melanomas. The 1986

17 Shields CL, Shields JA, De Potter P, et al. Treatment of nonresectable malignant iris tumours with custom designe plaque radiotherapy. Br $\mathcal{F}$ Ophthalmol 1995;79:306-12. 